PROCEEDINGS OF THE

AMERICAN MATHEMATICAL SOCIETY

Volume 132, Number 3, Pages 865-874

S 0002-9939(03)07280-0

Article electronically published on October 8, 2003

\title{
EXTENSION OF POSITIVE DEFINITE FUNCTIONS FROM SUBGROUPS OF NILPOTENT LOCALLY COMPACT GROUPS
}

\author{
EBERHARD KANIUTH
}

(Communicated by N. Tomczak-Jaegermann)

\begin{abstract}
We investigate the problem of when closed subgroups $H$ of a nilpotent locally compact group $G$ have the property that every continuous positive definite function on $H$ extends to some such function on $G$.
\end{abstract}

\section{INTRODUCTION}

Let $G$ be a locally compact group and $H$ a closed subgroup of $G$. Following the terminology of 17, we call $H$ an extending subgroup of $G$ if every continuous positive definite function on $H$ extends to some continuous positive definite function on $G$. This is equivalent to requiring that the restriction mapping $B(G) \rightarrow B(H)$ be surjective, where $B(-)$ denotes the Fourier-Stieltjes algebras (cf. [5]). We say that $G$ has the extension property when each closed subgroup of $G$ is extending. These properties have been studied by several authors [2], [3, 66, [9, [10, [16, [17]. Fundamental to all this has been Douady's observation (see [5, p. 204] and [12, (34.28)]) that if $A$ is an abelian closed normal subgroup of a locally compact group $G$ and $\chi$ is a character of $A$, then $\chi$ extends to some continuous positive definite function on $G$ only if the stabiliser $G_{\chi}=\left\{x \in G: \chi\left(x^{-1} a x\right)=\chi(a)\right.$ for all $\left.a \in A\right\}$ is open in $G$.

We mention some of the most significant results that have been obtained so far. It was independently shown by Henrichs $[9]$ and Cowling and Rodway 3$]$ that a closed subgroup $H$ of $G$ is extending if $G$ possesses small $H$-invariant neighbourhoods of the identity. In particular, every SIN-group, i.e., group with small conjugation invariant neighbourhoods of the identity, has the extension property. Conversely, a connected group $G$ has the extension property only if it is a SIN-group (equivalently, being connected, $G$ is the direct product of a vector group and a compact group) [3, Corollary 2]. This latter result can also directly be deduced from [11, Theorem 2 ]. On the other hand, in 10 an example is given of a 2-step solvable compactly generated locally compact group that has the extension property and nevertheless fails to be a SIN-group.

The preceding results naturally lead to specifically investigate the extension properties for nilpotent locally compact groups. In this paper we prove first that if $G$

Received by the editors November 14, 2002.

2000 Mathematics Subject Classification. Primary 43A35; Secondary 22E25.

(C)2003 American Mathematical Society 
is a nilpotent locally compact group that has the extension property and is either compactly generated or compact-free, then $G$ has to be a SIN-group (Theorem 2.5 and Corollary 2.3). However, the most intricate result that we have been able to obtain is that under the weaker hypothesis that $G$ is a nilpotent group having an almost connected open normal subgroup, requiring the extension property to hold for $G$ forces $G$ to possess at least one compact invariant neighbourhood of the identity (Theorem 2.6).

Turning to a special class of simply connected nilpotent Lie groups, we address in Section 3 the question of which closed subgroups are extending. Let $G_{n}, n \geq 3$, be one of the groups in the series of "threadlike" nilpotent Lie groups. We prove that the nontrivial extending subgroups of $G_{n}$ are precisely those closed subgroups that are topologically isomorphic to either $\mathbb{R}$ or $\mathbb{Z}$ (Theorem 3.6).

\section{Preliminaries}

We have to introduce some notation. For any group $G$, let $Z_{1}(G) \subseteq Z_{2}(G) \subseteq$ ... be the ascending central series of $G$, that is, $Z_{1}(G)$ is the centre of $G$ and $Z_{j+1}(G) / Z_{j}(G)$ equals the centre of $G / Z_{j}(G)$. If $G$ is a locally compact group and $A$ and $B$ are subsets of $G,[A, B]$ will denote the closed subgroup generated by the set of all commutators $[a, b]=a b a^{-1} b^{-1}, a \in A, b \in B$. Also, $G_{0}$ will denote the connected component of the identity. Finally, the term character means a continuous homomorphism from $G$ into the circle group $\mathbb{T}$.

We let $P(G)$ be the set of all continuous positive definite functions on $G$ and $P^{1}(G)=\{\phi \in P(G): \phi(e)=1\}$. For basic properties of positive definite functions we refer to [4] and [12]. Moreover, for any convex set $C$, such as $P^{1}(G), \operatorname{ex}(C)$ will denote the set of extreme points of $C$.

In this section we provide two basic results on positive definite functions which will be used several times.

Lemma 1.1. Let $\phi \in P^{1}(G)$. Then

$$
\begin{aligned}
\frac{1}{2}|1-\phi([x, y])| \leq & 1-|\phi(x)|+1-|\phi(y)| \\
& +(1-|\phi(x)|)(1-|\phi(y)|) \\
& +(1-|\phi(x)|)^{1 / 2}(1-|\phi(y)|)^{1 / 2}
\end{aligned}
$$

for all $x, y \in G$.

Proof. Recall first that

$$
|\phi(a) \phi(b)-\phi(a b)|^{2} \leq\left(1-|\phi(a)|^{2}\right)\left(1-|\phi(b)|^{2}\right)
$$

for all $a, b \in G[12$ Theorem 32.4(v)]. Using this inequality several times, we obtain 
for $x, y \in G$,

$$
\begin{aligned}
|1-\phi([x, y])| \leq & 1-|\phi(x)|^{2}+|\phi(x)|^{2}\left(1-|\phi(y)|^{2}\right) \\
& +\left.|| \phi(x) \phi(y)\right|^{2}-\phi(x) \phi(y) \phi\left(x^{-1} y^{-1}\right)|+| \phi(x) \phi(y) \phi\left(x^{-1} y^{-1}\right) \\
& -\phi(x) \phi\left(y x^{-1} y^{-1}\right)|+| \phi(x) \phi\left(y x^{-1} y^{-1}\right)-\phi([x, y]) \mid \\
\leq & 2(1-|\phi(x)|)+2(1-|\phi(y)|)+|\phi(x) \phi(y)-\phi(x y)| \\
& +\left|\phi(y) \phi\left(x^{-1} y^{-1}\right)-\phi\left(y x^{-1} y^{-1}\right)\right|+\left|\phi(x) \phi\left(y x^{-1} y^{-1}\right)-\phi([x, y])\right| \\
\leq & 2(1-|\phi(x)|)+2(1-|\phi(y)|)+\left(1-|\phi(x)|^{2}\right)^{1 / 2}\left(1-|\phi(y)|^{2}\right)^{1 / 2} \\
& +\left(1-|\phi(y)|^{2}\right)^{1 / 2}\left(1-\left|\phi\left(x^{-1} y^{-1}\right)\right|^{2}\right)^{1 / 2} \\
& +\left(1-|\phi(x)|^{2}\right)^{1 / 2}\left(1-\left|\phi\left(y x^{-1} y^{-1}\right)\right|^{2}\right)^{1 / 2} \\
\leq & 2(1-|\phi(x)|)+2(1-|\phi(y)|)+\left(1-|\phi(x)|^{2}\right)^{1 / 2}\left(1-|\phi(y)|^{2}\right)^{1 / 2} \\
& +2(1-|\phi(y)|)^{1 / 2}+2(1-|\phi(x)|)^{1 / 2}
\end{aligned}
$$

as claimed.

For any group $G$ and $x \in G$, we shall denote by $c(x)$ the mapping from $G$ into $G$ defined by $c(x) y=[x, y], y \in G$.

Corollary 1.2. Let $\phi \in P^{1}(G)$ and $y \in G$ such that $|\phi(y)|=1$. Given $m \in \mathbb{N}$ and $\epsilon>0$, there exists $\delta>0$ such that

$$
\left|1-\phi\left(c(x)^{m} y\right)\right| \leq \epsilon
$$

for all $x \in G$ with $1-|\phi(x)| \leq \delta$.

Proof. The statement holds for $m=1$ since, by Lemma 1.1,

$$
|1-\phi([x, y])| \leq 2\left(1-|\phi(x)|+(1-|\phi(x)|)^{1 / 2}\right) .
$$

Now, assuming the statement to hold for $m$, it follows for $m+1$, since

$$
\begin{gathered}
\frac{1}{2}\left|1-\phi\left(c(x)^{m+1} y\right)\right| \leq 1-|\phi(x)|+1-\left|\phi\left(c(x)^{m} y\right)\right| \\
+(1-|\phi(x)|)\left(1-\left|\phi\left(c(x)^{m} y\right)\right|\right)+(1-|\phi(x)|)^{1 / 2}+\left(1-\left|\phi\left(c(x)^{m} y\right)\right|\right)^{1 / 2} \\
\leq 1-|\phi(x)|+\left|1-\phi\left(c(x)^{m} y\right)\right|+(1-|\phi(x)|)\left|1-\phi\left(c(x)^{m} y\right)\right| \\
+(1-|\phi(x)|)^{1 / 2}+\left|1-\phi\left(c(x)^{m} y\right)\right|^{1 / 2}
\end{gathered}
$$

by Lemma 1.1 again.

\section{THE EXTENSION PROPERTY}

We start this section with two lemmas which turn out to be fundamental when dealing with nilpotent locally compact groups.

Lemma 2.1. Let $G$ be a locally compact group and $H$ a closed subgroup of $G$. Suppose that every character of $H$ extends to some function in $P(G)$. Then

$$
H \cap\left[H, Z_{2}(G)_{0}\right] \subseteq[H, H] .
$$

In particular, if $G$ is connected and 2-step nilpotent, then $H \cap[H, G]=[H, H]$. 
Proof. Suppose there exists $h_{0} \in H \cap\left[H, Z_{2}(G)_{0}\right]$ such that $h_{0} \notin[H, H]$. There exists a character $\chi$ of $H$ such that $\chi\left(h_{0}\right) \neq 1$. By hypothesis, there exists $\phi \in$ $P(G)$ such that $\left.\phi\right|_{H}=\chi$. By [10, Proposition 2, p. 275], $\phi$ can be chosen in $\operatorname{ex}\left(P^{1}(G)\right)$. Then $\left.\phi\right|_{Z_{1}(G)}$ is a character of $Z_{1}(G)$. Indeed, if $\pi_{\phi}$ denotes the cyclic representation defined by $\phi$, then $\pi_{\phi}$ is irreducible and hence $\left.\pi_{\phi}\right|_{Z_{1}(G)}$ is a multiple of a 1-dimensional representation.

Since $|\phi(h)|=1$ for all $h \in H$, we have (Lemma 1.1)

$$
\frac{1}{2}|1-\phi([h, x])| \leq 1-|\phi(x)|+(1-|\phi(x)|)^{1 / 2}
$$

for all $x \in G$ and $h \in H$. Thus there exists an open neighbourhood $V$ of the identity in $G$ such that $|1-\phi([h, x])|<\sqrt{3}$ for all $x \in V$ and $h \in H$.

Now, let $x \in V \cap Z_{2}(G)$. Then the map $h \rightarrow[h, x]$ is a homomorphism from $H$ into $Z_{1}(G)$. Since $\phi_{\mid Z_{1}(G)}$ is a character, it follows that the set $\Gamma_{\chi}=\{\phi([h, x]): h \in$ $H\}$ is a subgroup of $\mathbb{T}$ with the property that $|z-1|<\sqrt{3}$ for all $z \in \Gamma_{\chi}$. It is well known that this implies that $\Gamma_{\chi}=\{1\}$. Finally, since $x \rightarrow[h, x]$ is a homomorphism from $Z_{2}(G)$ into $Z_{1}(G)$, we conclude that $\phi([h, x])=1$ for all $h \in H$ and all $x$ in the open subgroup of $Z_{2}(G)$ generated by $V \cap Z_{2}(G)$. Therefore, $\phi(y)=1$ for all $y \in\left[H, Z_{2}(G)_{0}\right]$. Since $h_{0} \in\left[H, Z_{2}(G)_{0}\right]$, we have reached the contradiction $1=\phi\left(h_{0}\right)=\chi\left(h_{0}\right)$.

Lemma 2.2. Let $G$ be a semidirect product $G=A \ltimes N$, where $N$ is abelian, connected and contained in $Z_{m}(G)$ for some $m \in \mathbb{N}$. If $G$ has the extension property, then $N$ is contained in the centre of $G$.

Proof. We prove the lemma by induction on $m$. Thus, let $N \subseteq Z_{m+1}(G)$ and suppose that if $H=A \ltimes M$ is such that $H$ has the extension property and $M$ is abelian and connected and $M \subseteq Z_{m}(H)$, then $M$ is central in $H$.

Let $M=[A, N]$ and $H=A \ltimes M$. Then the induction hypothesis applies to $H$ since $M \subseteq Z_{m}(H)$. Thus $M$ is contained in the centre of $H$, and since $N$ is abelian, $M$ is even central in $G$. Now, $H=A M$ is an extending subgroup of $G$. Since $N$ is connected and $N \subseteq Z_{2}(G)$, Lemma 2.1 implies that

$$
[A, N]=A M \cap[A, N] \subseteq H \cap\left[H, Z_{2}(G)_{0}\right] \subseteq[H, H]=[A, A] .
$$

This shows that $[A, N]=\{e\}$ since $A \cap N=\{e\}$. Therefore, $N$ is central in $G$ since $N$ is abelian.

Let $H$ be a closed subgroup of a locally compact group $G$. Recall from 8 , that $G$ is said to have small $H$-invariant neighbourhoods $\left(G\right.$ belongs to the class $\left[\mathrm{SIN}_{H}\right)$ if $G$ has a neighbourhood basis $\mathcal{V}$ of the identity such that $h^{-1} V h=V$ for all $V \in \mathcal{V}$ and $h \in H$. If $G \in[\mathrm{SIN}]_{G}$, then $G$ is called a SIN-group. We let [SIN] denote the class of all SIN-groups. A connected group is a SIN-group if and only if it is a direct product of a vector group and a compact group [4, Theorem 16.4.6]. SIN-groups and their structure have been extensively studied by Grosser and Moskowitz [8].

Recall that a connected Lie group has the extension property only if it is a SINgroup. This easily extends to almost connected groups. Indeed, such a group $G$ is a projective limit of Lie groups $G_{\iota}=G / K_{\iota}$ [18, (4.6)], and each $\left(G_{\iota}\right)_{0}$ has the extension property. Thus $\left(G_{\iota}\right)_{0}$ is a SIN-group and so is $G_{\iota}$ since $\left(G_{\iota}\right)_{0}$ is of finite index in $G_{\iota}$. Now, it is easily verified that a projective limit of SIN-groups is again a SIN-group. 
For a locally compact group $G$, let $G^{c}$ denote the set of all compact elements of $G$ (that is, elements that generate a relatively compact subgroup). $G$ is said to be compact-free if $G^{c}=\{e\}$. Suppose that $G$ is nilpotent. Then $G^{c}$ is a closed (normal) subgroup of $G$ [7, Corollary 3.5.1 and Lemma 3.8] and $G / G^{c}$ is compactfree. If $G$ is compactly generated, then $G^{c}$ is even compact [7, Theorem 9.7]. When $G$ is discrete, $G^{c}$ is just the set of elements of finite order, usually denoted $G^{t}$, the torsion subgroup of $G$, and $G$ is called torsion-free if $G^{t}=\{e\}$.

We now study nilpotent groups having the extension property.

Corollary 2.3. Let $G$ be a compact-free nilpotent locally compact group. If $G$ has the extension property, then the centre of $G$ is open in $G$.

Proof. The connected component $G_{0}$ is open in $G$ and $G / G_{0}$ is torsion-free. Since $G_{0}$ has the extension property, $G_{0}=V \times K$, where $V$ is a vector group and $K$ is a compact group. Now $K$, being connected and nilpotent, is abelian. Consider any element $a \in G \backslash G_{0}$, and let $A$ be the subgroup generated by $a$. Since $G_{0}$ is open and $G / G_{0}$ is torsion-free, $A \cap G_{0}=\{e\}$ and hence $A G_{0}=A \ltimes G_{0}$. Since $G_{0}$ is abelian and $G_{0} \subseteq Z_{m}(G)$ and since $A \ltimes G_{0}$ has the extension property, an application of Lemma 2.2 yields that $G_{0}$ commutes with $a$. Since $a \in G \backslash G_{0}$ was arbitrary and $G_{0}$ is abelian and open, we conclude that the centre of $G$ is open.

Lemma 2.4. Let $G$ be a locally compact group and suppose that $G_{0}$ is open in $G$ and contained in $Z_{m}(G)$ for some $m \in \mathbb{N}$. Moreover, suppose that $G / G_{0}$ has a finite normal subgroup $M$ such that $\left(G / G_{0}\right) / M$ is torsion-free. If $G$ has the extension property, then $G$ is a SIN-group.

Proof. Since $G_{0}$ has the extension property, $G_{0}=V \times K$ where $K$ is a compact group and $V$ is a vector group. $K$ being connected and nilpotent, $K$ is abelian. Let $N \subseteq G$ denote the pullback of $M$.

Since $G_{0}$ is abelian and $G_{0} \subseteq Z_{m}(G)$, and since $G / N$ is torsion-free, an application of Lemma 2.2 (compare the proof of Corollary 2.3) yields that $G_{0}$ commutes with $a$ for each $a \in G \backslash N$. Since $N / G_{0}$ is finite, it follows that $G$ acts as a finite group of inner automorphisms on $G_{0}$. Since $G_{0}$ is open, this shows that $G$ is a SIN-group.

We are now ready for the two main results of this section.

Theorem 2.5. Let $G$ be a compactly generated nilpotent locally compact group. Then $G$ has the extension property (if and) only if $G$ is a SIN-group.

Proof. Since $G$ is compactly generated, it is a projective limit of Lie groups $G_{\alpha}=$ $G / K_{\alpha}, \alpha \in A$ [13]. Thus, since a projective limit of SIN-groups is a SIN-group and each $G_{\alpha}$ has the extension property, we can assume that $G$ is a Lie group. Then $G_{0}$ is open in $G$ and $G / G_{0}$ is a finitely generated nilpotent group. Let $N \subseteq G$ denote the pullback of the torsion subgroup $\left(G / G_{0}\right)^{t}$ of $G / G_{0}$. Then $N / G_{0}$ is finite since $\left(G / G_{0}\right)^{t}$ is finite, and $G / N$ is torsion-free. Lemma 2.4 now shows that $G$ is a SIN-group.

Theorem 2.6. Let $G$ be a nilpotent locally compact group and suppose that $G$ possesses an almost connected open normal subgroup. If $G$ has the extension property, then $G$ has a compact normal subgroup $K$ such that $G / K$ is a SIN-group. 
Proof. By hypothesis there exists an open normal subgroup $M$ of $G$ such that $M / G_{0}$ is compact. Since $M$ has the extension property, $M$ is a SIN-group. Let $N$ denote the subgroup of $M$ consisting of all elements $x \in M$ with the property that the conjugacy class of $x$ in $M$ is relatively compact. Then $N$ is an open normal subgroup of $G$ 8, and $N \supseteq G_{0}$, whence $M / N$ is finite.

By the structure theorem for SIN-groups with relatively compact conjugacy classes [8, Theorem 4.6], $N=V \times K$, where $V$ is a vector group and $K$ has a compact open subgroup. Since $N$ is almost connected, $K$ itself has to be compact. Clearly, $K=N^{c}$ is normal in $G$. Since $G / K$ has the extension property, after passing to $G / K$, we can assume that $G$ contains an open normal vector group $V$. Let $\operatorname{Aut}(V)$ denote the group of all topological automorphisms of $V$, equipped with its natural topology (see [12 Section 26]). If $V=\mathbb{R}^{d}$, then $\operatorname{Aut}(V)$ is topologically isomorphic with $G L(d, \mathbb{R})$.

Let $\alpha: x \rightarrow \alpha_{x}$ denote the homomorphism from $G$ into $\operatorname{Aut}(V)$ given by $\alpha_{x}(v)=$ $x^{-1} v x$ for $v \in V$. Let $H$ be the open subgroup of $G$ specified by $H / V=(G / V)^{t}$. Since $G$ has the extension property and $G / H$ is torsion-free, as has been seen in the proof of Corollary 2.3, $\alpha_{x}$ is the identity of $\operatorname{Aut}(V)$ for every $x \in G \backslash H$. Thus $\alpha(G)=\alpha(H)$ is a torsion subgroup of $\operatorname{Aut}(V)$. Let $\Gamma$ be the closure of $\alpha(H)$ in $\operatorname{Aut}(V)$. Since $\operatorname{Aut}(V)$ is a Lie group, $\Gamma_{0}$ is open in $\Gamma$ and hence $\Gamma=\alpha(H) \Gamma_{0}$. In particular, $\Gamma / \Gamma_{0}$ is a torsion group. Also, since $\Gamma_{0}$ is open in $\Gamma, \alpha(H) \cap \Gamma_{0}$ is dense in $\Gamma_{0}$. Hence $\Gamma_{0}$ is a connected nilpotent group with $\Gamma_{0}^{t}$ dense in $\Gamma_{0}$. Since $\Gamma_{0}^{c}$ is compact, it follows that $\Gamma_{0}$ is compact.

Since $\operatorname{Aut}(V)$ is a connected Lie group, $\Gamma_{0}$ is contained in a maximal compact subgroup $C$ of $\operatorname{Aut}(V)$. Define an ascending sequence of subgroups $\Gamma_{0} \subseteq \Gamma_{1} \subseteq \ldots$ of $\Gamma$ by $\Gamma_{1} / \Gamma_{0}=Z_{1}\left(\Gamma / \Gamma_{0}\right)$ and $\Gamma_{j+1} / \Gamma_{j}=Z_{1}\left(\Gamma / \Gamma_{j}\right)$ for $j \geq 1$. We prove by induction that $\Gamma_{j} \subseteq C$. Let $F$ be any subgroup of $\Gamma_{j+1}$ such that $F \supseteq \Gamma_{j}$ and $F / \Gamma_{j}$ is finite. Assuming that $\Gamma_{j} \subseteq C$, we have that $F C$ is a compact group, whence $F C=C$. Since $\Gamma_{j+1} / \Gamma_{j}$ is locally finite, we conclude that $\Gamma_{j+1} \subseteq C$. Since $\Gamma$ is nilpotent, it follows that $\Gamma \subseteq C$. Thus $\alpha(H)$ is a relatively compact subgroup of $\operatorname{Aut}(V)$. This proves that $V \in[\mathrm{SIN}]_{G}$, whence $G \in[\mathrm{SIN}]$ since $V$ is open in $G$.

Using Lemma 2.2 it is easy to see that Theorem 2.6 generalizes Corollary 2.3. However, the proof of Theorem 2.6 is so much more involved that it is justified to give the simple proof in the situation of Corollary 2.3.

An essential reason for why we are unable to treat general nilpotent locally compact groups is that, at least so far, totally disconnected groups seem to be inaccessible. We believe that, under the hypothesis of Theorem 2.6, even $G$ itself has to be a SIN-group.

Recently, a certain separation property of positive definite functions has been studied [14, 15]. A locally compact group $G$ is said to have the separation property if, given any closed subgroup $H$ of $G$ and $x \in G \backslash H$, there exists $\phi \in P(G)$ such that $\phi(x) \neq 1$ and $\phi(h)=1$ for all $h \in H$. It is worth mentioning that, although at first glance the extension and the separation properties seem to be unrelated, the results obtained for almost connected and for nilpotent groups are similar. However, the two properties are not equivalent in general. For instance, the semidirect product group $\mathbb{Z} \ltimes \mathbb{R}$, where $n \in \mathbb{Z}$ acts on $\mathbb{R}$ by multiplication with $2^{n}$, has the extension property [10], but not the separation property. 


\section{EXTENDING SUBGROUPS}

As pointed out in the introduction, if $H$ is a closed subgroup of a locally compact group $G$ such that $G \in[\mathrm{SIN}]_{H}$, then $H$ is an extending subgroup. This was shown in [9, 2.11. Korollar] and, although not stated there in this generality, 3. Theorem $2]$. In particular, compact and central subgroups are extending. For connected groups $G$, the condition that $G \in\left[\mathrm{SIN}_{H}\right.$ can be very restrictive. For instance, if $G$ is a connected nilpotent group and $H$ a closed subgroup of $G$, then $G \in[\mathrm{SIN}]_{H}$ forces $H$ to be contained in the centre of $G$ [15. Proposition 2.6]. As is to be expected, there are many other extending subgroups. However, for a given $G$, to determine all extending subgroups appears to be quite difficult.

In this section, for the so-called "threadlike" simply connected nilpotent Lie groups, we characterize all the extending subgroups up to topological isomorphism.

Lemma 3.1. Let $H$ and $N$ be closed subgroups of $G$ such that $N$ is normal, $H \cap N=$ $\{e\}, H N$ is closed and $H$ is topologically isomorphic to $H N / N$. Moreover, suppose that $H N / N$ is contained in the centre of $G / N$. Then $H$ is an extending subgroup.

Proof. Let $\varphi \in P(H)$ and define $\psi \in P(H N / N)$ by $\psi(h N)=\varphi(h), h \in H$. Since $H N / N$ is contained in the centre of $G / N, \psi$ extends to some $\rho \in P(G / N)$. With $q: G \rightarrow G / N$ the quotient homomorphism, we have that $\phi=\rho \circ q \in P(G)$ and $\phi(h)=\varphi(h)$ for all $h \in H$.

Let $G$ be a connected and simply connected nilpotent Lie group, and let $H$ and $N$ be closed subgroups of $G$. We shall use the fact that if $N$ is connected and normal, then $H N$ is closed in $G$ (cf. [1, Lemma 1.1]). This implies, with $q$ denoting the quotient homomorphism from $G$ onto $G / N$, that $q(H)$ is closed in $G / N$.

Corollary 3.2. Let $G$ be a 2-step nilpotent connected and simply connected Lie group, and let $H$ be an abelian closed subgroup of $G$. Then $H$ is extending if and only if $H \cap[H, G]=\{e\}$.

Proof. The necessity of the condition follows from Lemma 2.1. Conversely, let $N=[H, G]$ and suppose that $H \cap N=\{e\}$. Then $H N$ is a closed subgroup of $G$ and, since $H \cap N=\{e\}, H$ is topologically isomorphic to $H N / N$. Moreover, $H N / N$ is contained in the centre of $G / N$. Thus $H$ is extending by Lemma 3.1.

Lemma 3.3. Let $G$ be a connected and simply connected nilpotent Lie group and $H$ a closed subgroup that is topologically isomorphic to either $\mathbb{R}$ or $\mathbb{Z}$. Then $H$ is extending.

Proof. Let $\mathfrak{g}$ denote the Lie algebra of $G$, exp $: \mathfrak{g} \rightarrow G$ the exponential map and $\mathfrak{z}_{0}=\{0\} \subseteq \mathfrak{z}_{1} \subseteq \ldots$ the ascending central series of $\mathfrak{g}$. Suppose first that $H$ is isomorphic to $\mathbb{R}$ and let $H=\exp \mathfrak{h}$ and $Z_{j}=\exp \mathfrak{z}_{j}, j=0,1, \ldots$. Since $\mathfrak{h}$ is 1dimensional, $\mathfrak{h} \cap \mathfrak{z}_{j} \neq\{0\}$ implies that $\mathfrak{h} \subseteq \mathfrak{z}_{j}$. Let $j$ be minimal such that $\mathfrak{h} \subseteq \mathfrak{z}_{j+1}$. Then $\mathfrak{h} \cap \mathfrak{z}_{j}=\{0\}$, and hence $H \cap Z_{j}=\{e\}$. Moreover, $H Z_{j}$ is closed in $G$, $H Z_{j} / Z_{j} \subseteq Z_{j+1} / Z_{j}$ and, since $G$ is $\sigma$-compact, $H$ is topologically isomorphic to $H Z_{j} / Z_{j}$. Lemma 3.1 shows that $H$ is extending. Finally, if $H$ is isomorphic to $\mathbb{Z}$, there exists a closed subgroup $L$ isomorphic to $\mathbb{R}$ and containing $H$. Since $L$ is extending, so is $H$. 
For $n \geq 3$, let $\mathfrak{g}_{n}$ be the $n$-dimensional nilpotent Lie algebra with basis $X_{1}, \ldots$, $X_{n}$ and nontrivial Lie brackets

$$
\left[X_{n}, X_{n-1}\right]=X_{n-2}, \ldots,\left[X_{n}, X_{2}\right]=X_{1} .
$$

Then $\mathfrak{g}_{n}$ is $(n-1)$-step nilpotent and a semidirect product of $\mathbb{R} X_{n}$ with the abelian ideal $\mathfrak{a}_{n}=\sum_{j=1}^{n-1} \mathbb{R}_{j}$. Let $G_{n}$ be the associated simply connected Lie group. Note that $G_{3}$ is just the Heisenberg group. The groups $G_{n}$ are often referred to as the "threadlike" nilpotent Lie groups. Of course, $G_{n}$ is a semidirect product $G_{n}=$ $\mathbb{R} \ltimes \mathbb{R}^{n-1}$, and we shall write elements of $G_{n}$ as pairs $(t, u)$, say, where $t \in \mathbb{R}$ and $u \in \mathbb{R}^{n-1}$. Also, we identify $\mathbb{R}^{n-1}$ with $\{0\} \times \mathbb{R}^{n-1}$ and $\mathbb{R}$ with $\mathbb{R} \times\{0\}$.

In the sequel, for a closed subgroup $H$ of $G$, the term proper means that $H \neq G$ and $H \neq\{e\}$. We are going to prove that in $G_{n}$ there are no proper extending subgroups other than those shown to be extending in Lemma 3.3.

Lemma 3.4. Let $H$ be a closed nontrivial subgroup of $G_{n}$ that is contained in $\mathbb{R}^{n-1}$. Suppose that every character of $H$ extends to some function in $P^{1}\left(G_{n}\right)$. Then $H$ is topologically isomorphic to either $\mathbb{R}$ or $\mathbb{Z}$.

Proof. We can assume that $H \nsubseteq Z_{1}\left(G_{n}\right)$. Let $m \in \mathbb{N}$ be minimal such that $H \subseteq$ $Z_{m+1}\left(G_{n}\right)$. Then, with the notation introduced before Corollary 1.2 ,

$$
\{e\} \neq c(x)^{m} H=\left\{c(x)^{m} y: y \in H\right\} \subseteq Z_{1}\left(G_{n}\right)
$$

for each nontrivial element $x$ of $\exp \left(\mathbb{R} X_{n}\right)$.

Let $\chi$ be any character of $H$. By hypothesis and [10, Proposition 2, p. 275], there exists $\phi \in \operatorname{ex}\left(P^{1}\left(G_{n}\right)\right)$ such that $\left.\phi\right|_{H}=\chi$. Since $|\phi(y)|=1$ for all $y \in H$, by Corollary 1.2 there exists a neighbourhood $V$ of the identity in $\exp \left(\mathbb{R} X_{n}\right)$ such that

$$
\left|1-\phi\left(c(x)^{m} y\right)\right|<\sqrt{3}
$$

for all $x \in V$ and $y \in H$. Now, since $\left[G_{n}, G_{n}\right] \subseteq \mathbb{R}^{n-1},[x, a b]=[x, a][x, b]$ for all $x \in G_{n}$ and $a, b \in \mathbb{R}^{n-1}$. Thus the mapping $y \rightarrow c(x)^{m} y$ from $H$ into $Z_{1}\left(G_{n}\right)$ is a homomorphism. Since $\left.\phi\right|_{Z_{1}\left(G_{n}\right)}$ is a character, the function $y \rightarrow \phi\left(c(x)^{m} y\right)$ from $H$ into $\mathbb{T}$ is a homomorphism. Hence, for each $x \in V$, the set

$$
\Gamma_{x}=\left\{\phi\left(c(x)^{m} y\right): y \in H\right\}
$$

is a subgroup of $\mathbb{T}$ with the property that $|\lambda-1|<\sqrt{3}$ for all $\lambda \in \Gamma_{x}$. This implies that $\Gamma_{x}=\{1\}$ for each $x \in V$. Thus there exists $y \in H$ such that $c(V)^{m} y$ is nontrivial and contained in the kernel of the character $\left.\phi\right|_{Z_{1}\left(G_{n}\right)}$. This shows that the kernel of $\left.\phi\right|_{Z_{1}\left(G_{n}\right)}$ cannot be discrete. Since $Z_{1}\left(G_{n}\right)=\mathbb{R}$, it follows that $\phi(z)=1$ for all $z \in Z_{1}\left(G_{n}\right)$.

Since every character of $H$ extends to some such $\phi$, this shows in particular that $H \cap Z_{1}\left(G_{n}\right)=\{e\}$. Therefore $H$ is topologically isomorphic to the quotient group $H Z_{1}\left(G_{n}\right) / Z_{1}\left(G_{n}\right)$, and since each such $\phi$ is identically one on $Z_{1}(G)$, we conclude from what we have shown above that every character of $H Z_{1}\left(G_{n}\right) / Z_{1}\left(G_{n}\right)$ extends to some element of $P^{1}\left(G_{n} / Z_{1}\left(G_{n}\right)\right)$.

We now proceed by induction on $n$. If $n=3$, then $H$ is isomorphic to some subgroup of $\mathbb{R}^{2} / Z_{1}\left(G_{3}\right)=\mathbb{R}$. Suppose the lemma holds for some $n-1, n \geq 4$. Then, since $G_{n} / Z_{1}\left(G_{n}\right)$ is isomorphic with $G_{n-1}$, the induction hypothesis yields that $H Z_{1}\left(G_{n}\right) / Z_{1}\left(G_{n}\right)$ is topologically isomorphic to either $\mathbb{R}$ or $\mathbb{Z}$, and hence the same is true for $H$. 
Lemma 3.5. Let $H$ be a proper closed subgroup of $G_{n}$ such that $H$ is not contained in $\mathbb{R}^{n-1}$. If $H$ is extending, then $H$ is isomorphic to $\mathbb{R}$ or $\mathbb{Z}$.

Proof. Let $H$ be any closed subgroup of $G_{n}$. We first observe the following two facts:

(i) if $H$ is not contained in $\mathbb{R}^{n-1}$, then $\left[H, Z_{2}\left(G_{n}\right)\right]=Z_{1}\left(G_{n}\right)$;

(ii) if $H_{0}$ is not contained in $\mathbb{R}^{n-1}$ and $H$ is not isomorphic to $\mathbb{R}$, then $H \supseteq$ $Z_{1}\left(G_{n}\right)$.

While (i) is obvious, (ii) can be seen as follows. Let $q: G_{n} \rightarrow G_{n} / \mathbb{R}^{n-1}$ denote the quotient homomorphism. Since $q\left(H_{0}\right)=\mathbb{R}$, for each $t \in \mathbb{R}$ there exists $u_{t} \in$ $\mathbb{R}^{n-1}$ such that $\left(t, u_{t}\right) \in H$. Moreover, since $H$ is not isomorphic to $\mathbb{R}$ and $q\left(H_{0}\right)=$ $\mathbb{R}$, there exists a nonzero $v \in \mathbb{R}^{n-1}$ such that $(0, v) \in H$. Let $1 \leq r \leq n-1$ be minimal with the property that $(0, v) \in Z_{r}\left(G_{n}\right)$. Then $c\left(t, u_{t}\right)^{r-1}(0, v) \in Z_{1}\left(G_{n}\right)$, and because $t$ is arbitrary the set of all these $(r-1)$-fold commutators generates $Z_{1}\left(G_{n}\right)$. This proves (ii).

We proceed to prove by induction on $n$ that if $H$ is a proper closed subgroup of $G_{n}$ such that $H_{0}$ is not contained in $\mathbb{R}^{n-1}$, then $H$ is isomorphic to $\mathbb{R}$. To start with, let $n=3$ and assume that $H$ fails to be isomorphic to $\mathbb{R}$. Then $H \supseteq Z_{1}\left(G_{3}\right)$ by (ii), and hence $H_{0}$ is normal in $G_{3}$. Moreover, $\operatorname{dim} H_{0}=2$ since $\mathbb{R}=Z_{1}\left(G_{3}\right) \subsetneq H_{0} \subsetneq G_{3}$. Thus $H_{0}$ is an abelian normal subgroup of $G_{3}$, and $H_{0}$ is extending since it is open in $G_{3}$. This contradicts Douady's result. Now let $n \geq 4$ and suppose the above statement holds for $n-1$. Let $H$ be a proper extending subgroup of $G_{n}$ such that $H_{0} \not \subset \mathbb{R}^{n-1}$ and, towards a contradiction, assume that $H$ is not isomorphic to $\mathbb{R}$. By (ii) again, $Z_{1}\left(G_{n}\right) \subseteq H$. Then $H / Z_{1}\left(G_{n}\right)$ is a proper extending subgroup of $G_{n} / Z_{1}\left(G_{n}\right)$, and $\left(H / Z_{1}\left(G_{n}\right)\right)_{0} \supseteq H_{0} / Z_{1}\left(G_{n}\right)$. Since $G_{n} / Z_{1}\left(G_{n}\right)$ is isomorphic to $G_{n-1}=\mathbb{R} \ltimes \mathbb{R}^{n-2}$ and $\left(H / Z_{1}\left(G_{n}\right)\right)_{0}$ is not contained in $\mathbb{R}^{n-2}$, the induction hypothesis yields that $H / Z_{1}\left(G_{n}\right)$ is isomorphic to $\mathbb{R}$. In particular, $H$ is abelian. Then, by (i) and Lemma 2.1,

$$
Z_{1}\left(G_{n}\right)=\left[H, Z_{2}\left(G_{n}\right)\right] \cap H=[H, H]=\{e\},
$$

a contradiction.

It remains to consider an extending subgroup $H$ of $G_{n}$ such that $H \nsubseteq \mathbb{R}^{n-1}$, but $H_{0} \subseteq \mathbb{R}^{n-1}$. Assume that $H$ is not isomorphic to either $\mathbb{R}$ or $\mathbb{Z}$. Let $\widetilde{H}$ denote the unique minimal connected subgroup of $G_{n}$ containing $H$ [19, Proposition 2.5]. Then $\operatorname{dim} \widetilde{H}=2$, whence $\widetilde{H}$ and hence $H$ is abelian. Also, $Z_{1}\left(G_{n}\right) \subseteq \widetilde{H}$ by (ii), since $\widetilde{H}$ is not contained in $\mathbb{R}^{n-1}$. It follows that $H \cap Z_{1}\left(G_{n}\right) \neq\{e\}$ since otherwise $H=H Z_{1}\left(G_{n}\right) / Z_{1}\left(G_{n}\right)$ is a closed subgroup of

$$
\widetilde{H} Z_{1}\left(G_{n}\right) / Z_{1}\left(G_{n}\right)=\widetilde{H} / \widetilde{H} \cap Z_{1}\left(G_{n}\right)=\widetilde{H} / Z_{1}\left(G_{n}\right)=\mathbb{R},
$$

which is a contradiction. Thus as above, Lemma 2.1 gives that

$$
\{e\} \neq H \cap Z_{1}\left(G_{n}\right)=H \cap\left[H, Z_{2}\left(G_{n}\right)\right]=[H, H]=\{e\} .
$$

This contradiction shows that $H$ is not isomorphic to $\mathbb{R}$ or $\mathbb{Z}$, and the proof is complete.

The following theorem is now an immediate consequence of Lemma 3.3, Lemma 3.4 and Lemma 3.5.

Theorem 3.6. Let $H$ be a proper closed subgroup of $G_{n}$. Then $H$ is an extending subgroup if and only if $H$ is topologically isomorphic to either $\mathbb{R}$ or $\mathbb{Z}$. 


\section{REFERENCES}

[1] M. B. Bekka and E. Kaniuth, Topological Frobenius properties for nilpotent groups, Math. Scand. 63 (1988), 282-296. MR 91a:22003

[2] L. N. Carling, On the restriction map of the Fourier-Stieltjes algebra $B(G)$ and $B_{p}(G)$, J. Funct. Anal. 25 (1977), 236-243. MR 56:16254

[3] M. Cowling and P. Rodway, Restrictions of certain function spaces to closed subgroups of locally compact groups, Pacific J. Math. 80 (1979), 91-104. MR 80i:43008

[4] J. Dixmier, $C^{*}$-algebras, North-Holland, Amsterdam, 1977. MR 56:16388

[5] P. Eymard, L'algèbre de Fourier d'un groupe localement compact, Bull. Soc. Math. France 92 (1964), 181-236. MR 37:4208

[6] V. Flory, Eine Lebesgue-Zerlegung und funktorielle Eigenschaften der Fourier-Stieltjes Algebra, Inaugural Dissertation, Universität Heidelberg, 1972.

[7] V. M. Gluskov, Locally nilpotent locally bicompact groups, Trudy Moscov. Mat. Obshch. 4 (1955), 291-332. (Russian) MR 17:281b

[8] S. Grosser and M. Moskowitz, Compactness conditions in topological groups, J. reine angew. Math. 246 (1971), 1-40. MR 44:1766

[9] R. W. Henrichs, Über Fortsetzung positiv definiter Funktionen, Math. Ann. 232 (1978), 131150. MR 58:2022

[10] R. W. Henrichs, On characters of subgroups, Indag. Math. 41 (1979), 273-281. MR 80i:43009

[11] R. W. Henrichs, On one-sided harmonic analysis, Proc. Amer. Math. Soc. 80 (1980), 627-630. MR 82e: 43003

[12] E. Hewitt and K. A. Ross, Abstract harmonic analysis. I, II, Springer-Verlag, Berlin, 1963/1970. MR 28:158, MR 41:7378

[13] K. H. Hofmann, J. R. Liukkonen and W. M. Mislove, Compact extensions of compactly generated nilpotent groups are pro-Lie, Proc. Amer. Math. Soc. 84 (1982), 443-448. MR 84e:22007

[14] E. Kaniuth and A. T. Lau, A separation property of positive definite functions on locally compact groups and applications to Fourier algebras, J. Funct. Anal. 175 (2000), 89-110. MR 2001m:43012

[15] E. Kaniuth and A. T. Lau, On a separation property of positive definite functions on locally compact groups, Math. Z. 243 (2003), 161-177.

[16] J. R. Liukkonen and W. M. Mislove, Symmetry in Fourier-Stieltjes algebras, Math. Ann. 217 (1975), 97-112. MR 54:8163

[17] J. R. McMullen, Extensions of positive-definite functions, Mem. Amer. Math. Soc. 117, American Mathematical Society, Providence, RI, 1972. MR 56:3573

[18] D. Montgomery and L. Zippin, Topological transformation groups, Interscience Publishers, New York, 1955. MR 17:383b

[19] M. S. Ragunathan, Discrete subgroups of Lie groups, Ergebnisse der Mathematik und ihrer Grenzgebiete, Band 68, Springer-Verlag, New York - Heidelberg, 1972. MR 58:22394a

Institut für Mathematik, Universität Paderborn, D-33095 Paderborn, Germany

E-mail address: kaniuth@math.uni-paderborn.de 